\title{
Vectores de expansión urbana y su interacción con los patrones socioeconómicos existentes en la ciudad de Santiago
}

Tomás Cox. Universidad de Chile, Santiago, Chile.

Ricardo Hurtubia. Pontificia Universidad Católica de Chile, Santiago, Chile.

RESUMEN | En el contexto de la expansión del Área Metropolitana de Santiago, realizada sin planes maestros y a partir de las iniciativas de desarrolladores inmobiliarios que deciden la localización y el tipo de proyectos por ejecutar (cumpliendo con ciertas condiciones), la segregación de la ciudad de Santiago se reproduce en los nuevos proyectos en el área periurbana. Este trabajo tiene como hipótesis que el precio de viviendas en un desarrollo inmobiliario nuevo se explica por su relación con otros proyectos existentes. Tal relación se mide a partir de los tiempos de viaje entre los proyectos, clasificados según su precio. Los resultados verifican que estos buscan vincularse con proyectos de su mismo tipo y también con los grupos socioeconómicos que se les asocian. Esto indica que si bien los nuevos desarrollos de un cierto nivel socioeconómico se pueden localizar en sectores de un nivel diferente, de todas formas mantienen relaciones con sus similares.

PALABRAS CLAVE | expansión urbana, mercado inmobiliario, segregación.

ABSTRACT In the context of the expansion of the metropolitan area of Santiago without master plans and based on real estate developers who decide the location and type of project to be developed (meeting certain conditions), the segregation of Santiago is reproduced in the new projects in the peri-urban area. The hypothesis of this investigation is that the unit price in a new housing development is explained by its relationship with other existing projects. The relation is measured considering travel times between projects, ranked by price. The results verify that projects seek proximity to the projects of the same type and with the socioeconomic groups to which they are associated. This indicates that while new developments aimed at a certain socioeconomic group can be located in other socioeconomic sectors, they do maintain relationships with their peers.

KEY WORDS | urban sprawl, real estate market, segregation. 


\section{Introducción}

Santiago es una ciudad que se expande rápidamente y en la que priman criterios de mercado con una laxa regulación del uso del suelo; por esto, comprender cómo se toman las decisiones de desarrollo y localización de proyectos inmobiliarios es fundamental para (1) indicar tendencias a futuro (sin cambios en marco regulatorio), y (2) para definir mecanismos e incentivos adecuados para un desarrollo territorial armónico y sustentable.

De acuerdo con la literatura, la decisión de localización residencial está fuertemente influenciada por la composición socioeconómica del sector (Hurtubia \& Bierlaire, 2014; Ioannides, 2004; Rodríguez, 2008; Sabatini, 2006). Sin embargo, a primera vista, se observa que las relaciones de segregación espacial se establecen a distintas escalas y no de manera uniforme en el espacio. En el presente trabajo se propone una metodología para cuantificar relaciones de segregación a diferentes escalas mostrando proximidades y separaciones entre grupos, las cuales permiten explicar o entender procesos de urbanización en el periurbano que no dependen de grupos preexistentes en el sector, sino de conexiones con grupos a escala regional. Un caso claro es el sector de Chicureo, el cual abordaremos más adelante. Esto implica una visión dinámica del territorio, en la cual los desarrolladores abordan la ciudad a partir de ejes de desarrollo, buscando conexiones con grupos que otorgan "estatus" y evitando sectores próximos a grupos de menores ingresos. El objetivo de esta investigación es cuantificar estas relaciones, resaltando un tipo de atributo que es importante considerar para modelar la localización residencial. Para esto se aborda la distribución de proyectos nuevos de casas en el espacio periurbano de Santiago, considerando los tiempos de viaje entre proyectos de diferentes precios, como también hacia distintos grupos socioeconómicos.

Este enfoque permite abordar el territorio como una entidad que, si bien en el caso de Santiago tiene una centralidad predominante (Rodríguez, 2012), también acoge relaciones entre múltiples destinos y, en muchos casos, referentes a relaciones sociales. En tal sentido, la metodología usada en este trabajo para medir la proximidad (tiempos de viaje promedio) permite calcular para cada localización de la región un promedio ponderado de los tiempos de viaje a un conjunto de destinos, y no solo a uno central. La ponderación se da por el peso ${ }^{1}$ que tiene cada uno de los destinos. Esta medida es más comprensiva que la medición a un único destino o al destino más cercano. No se considera una medida de tiempo o distancia al centro, ya que este destino puede cambiar y es más adecuado pensar en diversas centralidades con diferentes pesos.

Se busca con esta medición observar las diferencias en tiempos de viajes entre proyectos de igual y de diferentes tipos (separados en cinco grupos por precio UF/ $\left.\mathrm{m}^{2}\right)^{2}$, verificando si los proyectos similares buscan estar cerca entre ellos y lejanos a proyectos de otros precios. De la misma forma, se mide el tiempo de viaje desde los

1 Este peso se puede referir, por ejemplo, a la cantidad de puestos de trabajo (para accesibilidad al trabajo), categoría del hospital o cantidad de camas (para accesibilidad a salud), etcétera.

2 UF: Unidad de Fomento. Unidad de valor actualizada según inflación, que corresponde a 24.059,17 pesos chilenos (42,7 dólares) al día 27 de julio de 2014 . 
diferentes tipos de proyectos hacia hogares preexistentes de acuerdo con el Censo 2002, separados por Grupos Socioeconómicos (GSE) ${ }^{3}$, intentando verificar si los proyectos de mayor valor buscan localizarse cercanos a los hogares de altos ingresos y distantes de los de bajos ingresos.

Se considera el universo de proyectos inmobiliarios habitacionales (solo casas) en Santiago que se localizaron fuera del anillo Américo Vespucio, entre los años 2004 y 2013. Esto corresponde a un total de 89.422 unidades de vivienda distribuidos en 1.833 proyectos.

No es fácil cuantificar el peso relativo que tiene este tipo de urbanizaciones, pero es posible realizar algunas proyecciones. De acuerdo con las estimaciones demográficas del Instituto Nacional de Estadísticas (INE), la Región Metropolitana creció en 717.561 habitantes en el período 2004-2013.4 A su vez, si se considera un rango de entre 3 y 4 personas por vivienda (INE [2007] indica un promedio de 3,5 personas por hogar), se puede estimar que los proyectos estudiados han acogido un rango de 357.688 a 268.266 personas en un periodo similar, lo cual implica aproximadamente un $37,4 \%$ y $49,8 \%$ de la nueva oferta que tuvo que producirse. Es difícil cuantificar este impacto con precisión (por diferencias en el área de estudio, cambios en el tamaño de los hogares, estructura demográfica, etcétera), pero podemos asegurar que el peso de estos proyectos no es menor dentro de la oferta de la ciudad.

Graficando en el área de estudio los tiempos de viaje promedio a diferentes GSE y tipos de proyectos, se podrían distinguir los sectores que tenderían a acoger a futuros planes de diferentes rangos de precio, como también entender algunos de los motivos de la expansión residencial de la ciudad, como es la búsqueda de sectores con el perfil adecuado para cada proyecto.

\section{Expansión urbana y segregación}

Hay algunos elementos que son característicos del desarrollo en las ciudades latinoamericanas, siendo la segregación uno de los principales. De Ramón (2000) plantea que históricamente las élites han querido distanciarse, buscando más espacio y diferenciación. Este fenómeno ha producido una distribución segregada a gran escala, pero de todas formas con variaciones como las que se dan actualmente, disminuyendo en la microescala aunque aumentando en la macroescala, además de aumentar su "malignidad" (Sabatini, Cáceres \& Cerda, 2001). La decisión de localización residencial en Santiago depende en gran parte de la composición socioeconómica o el nivel de ingreso promedio del barrio. Análisis econométricos previos (Martínez, 1996) muestran que la decisión de localización residencial se explica (en promedio y aproximadamente) en un tercio por la accesibilidad a servicios, un tercio por las características de la vivienda y otro tercio por la composición socioeconómica del sector. En este fenómeno, las fuerzas de "atracción y repulsión" entre diferentes grupos socioeconómicos genera un complejo juego de desplazamientos

3 Los Grupos Socioeconómicos (GSE) son una clasificación realizada por la empresa de estudios de mercado Adimark (2004) y que se basa en el nivel educacional del jefe de hogar y las posesiones del hogar, a partir de información censal.

4 En http://www.ine.cl/canales/chile_estadistico/familias/demograficas_vitales.php. 
en la ciudad, en la cual hay grupos que desean vivir junto a sus "iguales" y otros que desean vivir junto a "los otros". Generalmente por "aspiracionalidad" (querer parecerse a los grupos socioeconómicos más altos), los grupos que intentan vivir junto a "los otros" son de menor GSE que "el otro".

Las dinámicas de segregación se configuran en conjunto con procesos de expansión. La industrialización en la ciudad latinoamericana produjo un éxodo rural de familias que se localizaron en la periferia, lo cual se potenció con una implementación de infraestructura vial y de comunicaciones que ha permitido el desarrollo de nuevos nodos periféricos (Bahr \& Borsdorf, 2005). Posteriormente, la liberalización de los mercados en Chile ha llevado a que la provisión de servicios y de vialidad pueda anticiparse o seguir rápidamente a este crecimiento urbano (Figueroa, 2004). Esta liberalización se ubica en un marco más general de globalización y apertura a capitales para proyectos y a una mayor motorización e ingresos para acceder a ellos (De Mattos, 1999).

La dinámica descrita produce espacios con complejas interacciones entre lo rural productivo, habitacional, servicios y subcentros. Tal entrelazamiento funcional ha sido estudiado desde el concepto de lo periurbano (Ávila, 2001), término difuso como el fenómeno que quiere estudiar, pero que sirve para denominar la interfaz entre lo urbano consolidado y lo rural.

\section{Formas de expansión habitacional}

La expansión no solo se ha producido mediante difusión continua, sino también por el desarrollo de antiguos y, sobre todo, nuevos satélites de Santiago.

Las condiciones normativas explican en gran medida los tipos de desarrollos que se han generado. El actual escenario se basa en una planificación por condiciones (normada en el Plan Regulador Metropolitano de Santiago de 1997 y 2003), según la cual las áreas de desarrollo son propuestas por los privados, incluso con la normativa y la infraestructura altamente influenciadas por las gestiones de inmobiliarias y propietarios de la tierra.

Gran parte del crecimiento se ha dado por agregación continua: la mancha urbana creció 12.049 hectáreas entre 1991 y 2000, siendo la mayor parte para destino habitacional (41\%) e industrial (23\%) (Ducci \& González, 2006); sin embargo, un cálculo que permita dimensionar el crecimiento del sistema metropolitano debe incluir las áreas en desarrollo que gravitan directamente sobre Santiago. Desde 2002 a 2013, solo en la provincia de Chacabuco se han construido 496 hectáreas en calidad de Zonas de Desarrollo Urbano Condicionado y hay todavía 6.062 hectáreas remanentes aprobadas en esta figura. Esto sin considerar las Áreas Urbanizables de Desarrollo prioritario (AUDP), que se anexan a localidades ya constituidas, y los Proyectos de Desarrollo Urbano Condicionado (PDUC), en tramitación en Pudahuel (1.187 ha más) (Transsa Consultores, 2013).

\section{Segregación desde la perspectiva espacio-temporal}

El estudio de las diferentes escalas de segregación que el desarrollo inmobiliario descrito produce es difícilmente captado por los índices tradicionales de segregación. Por ejemplo, el índice de disimilaridad de Duncan (Duncan \& Duncan, 1955) 
considera las proporciones de grupos por zonas, pero no el ordenamiento o relación espacial entre las ellas, por lo que puede medir segregación tan solo para una escala o nivel de agregación a la vez. Otros índices más comprensivos, como los recopilados por Massey y Denton (1988), también trabajan sobre zonas, indistintamente de la localización o separación entre ellas, salvo el índice de clusterización (que de todas formas trabaja sobre zonas estáticas, sin informar mayormente sobre relaciones a mayor escala).

Posteriormente se han propuesto medidas que abarquen diferentes escalas de segregación (Lee et al., 2008; Leckie, Pillinger, Jones \& Goldstein, 2012), como un fenómeno que se puede experimentar no solo en el barrio residencial (estáticamente), sino también en las actividades que realiza el individuo. Este enfoque se basa en conceptos de geografía temporal que consideran el prisma espacio-temporal de los sujetos (Hägerstrand, 1970), analizando sus trayectorias al trabajo y otras actividades, como también las dimensiones temporales de estos destinos (horas de apertura, etcétera) (Kwan, 2015; Palmer, 2013; Schnell \& Yoav, 2001; Wang \& Chai, 2012). Otros estudios integran a la medición de accesibilidad conceptos relacionados con la movilidad individual (Hannam, Sheller \& Urry, 2006), aplicando a ello un mayor sustento teórico, pero también con mayores complejidades en cuanto a información e implementación en políticas urbanas (Hine \& Mitchell, 2001).

Los autores señalados estudian la "experiencia de la segregación” desde el individuo, pero también es necesario comprender las dinámicas que generan las diferentes distribuciones de usos de suelo. Si bien se puede entender la segregación como un fenómeno que emerge descentralizadamente (Schelling, 1978), también hay políticas públicas y privadas que la estimulan o que la incorporan como parte del producto inmobiliario. En el contexto latinoamericano se ha estudiado el surgimiento de conjuntos habitacionales altamente homogéneos y que buscan diferenciarse del entorno, definidos como "conjuntos vallados" (Hidalgo, 2004), y que se expresan como proyectos identificables (por ejemplo, en la Región Metropolitana de Santiago, Lo Campino en Quilicura, Ciudad de los Valles en Pudahuel, Chamisero en Colina, Alto El Manzano en Tiltil, etcétera). Generalmente se asocia estas tipologías a conjuntos de altos ingresos y con viviendas de mayor valor. La separación de tales proyectos se manifiesta en límites materiales (muros, rejas) o semánticos (denominaciones, colores, materiales, pertenencias a comunas). El objetivo explícito de los límites materiales es la seguridad, y de los semánticos es el valor agregado de estas características (aprovechar el mejor equipamiento de una comuna de altos ingresos, mejor calidad de la construcción); sin embargo, existe un objetivo implícito o no declarado ("incómodo"), que es la separación respecto a otros grupos socioeconómicos, explicada en esta repulsión de grupos de más altos ingresos hacia los de menores ingresos, detallada más arriba. La tipología de "conjuntos vallados" se relaciona con proyectos habitacionales localizados en áreas de expansión, por lo que tienen la incertidumbre del entorno que se conformará a futuro.

Atkinson y Flint (2004) han estudiado este fenómeno en el Reino Unido, enfatizando las relaciones dinámicas de las Gated Communities con su entorno a mayor escala y su inserción en redes bien conectadas con otros nodos premium, pero poco relacionadas con su entorno inmediato. 


\section{La accesibilidad a GSE como variable explicativa del desarrollo habitacional en expansión}

Considerando el territorio como un sistema, la decisión de localización de un hogar en la ciudad no tiene relación exclusivamente con los atributos del entorno inmediato o barrio como una entidad aislada. El desarrollo habitacional del Área Metropolitana de Santiago (AMs) no puede ser explicado solo desde esa perspectiva, más aún si vemos los desarrollos de proyectos en sectores como Chicureo o El Carmen de Huechuraba. ${ }^{5}$

En áreas en las cuales el perfil del entorno no está todavía bien definido, los desarrolladores buscan "tomar posición" de forma estratégica para distanciar su proyecto de otros grupos socioeconómicos, pero también asegurarse de acercarlo a otros GSE deseados. Esto se vuelve crítico cuando se observa que la expansión urbana genera procesos crecientes de fragmentación espacial y social (Farber \& Li, 2013; Inostroza, Baur \& Csaplovics, 2013).

Para esta investigación se explora la variable de accesibilidad entre los proyectos de distintos precios, considerando que existen atracciones y repulsiones entre diferentes grupos socioeconómicos. A esto se suma el uso de los límites topográficos como forma de aislar los desarrollos, teniendo como hipótesis que los proyectos de mayor precio se ubican en localizaciones menos abiertas, con el objetivo de distanciarse de los de menor precio.

\section{Metodología}

A continuación se presentan las fuentes de información y su tratamiento, así como la metodología utilizada para medir tiempos de viaje.

\section{Proyectos inmobiliarios nuevos}

La base de datos de proyectos inmobiliarios fue levantada por las empresas Collect e Inciti, mediante la visita a la totalidad de los proyectos en venta en la Región Metropolitana entre 2004 y 2013. A partir de ella, de datos se construyó una base de 1.833 observaciones de etapas de proyectos, con un total de 89.422 unidades de viviendas nuevas y sus respectivos valores totales (UF) ${ }^{6}$ e Índice de Construcción (valor total dividido por superficie construida, expresado en $\mathrm{UF} / \mathrm{m}^{2}$ ). Se optó por considerar solo los proyectos de vivienda, dado que son los más representativos de la expansión urbana y son comparables entre sí. Salvo contados proyectos, se tomaron solo los aquellos que se ubican del Anillo Américo Vespucio hacia afuera, los cuales se consideran ubicados en áreas suburbanas o de expansión.

Las viviendas se dividieron en tipos (tabla 1) según su valor unitario de venta $\left(\mathrm{uF} / \mathrm{m}^{2}\right)$. La división se realizó mediante el método de Quiebres Naturales (Natural Breaks) de Jenks (1967), que busca, mediante iteraciones sucesivas sobre un rango

5 Estos sectores, representados en el mapa 1, muestran proyectos que están por sobre lo observado anteriormente en el sector, saliéndose del "cono de altos ingresos" (sector oriente de la ciudad), pero de todas formas bien conectados con estos barrios.

6 UF: Unidad de Fomento. Unidad de valor actualizada según inflación que corresponde a 24.059,17 pesos (42,7 dólares) al día 27 de julio de 2014. 
de observaciones, los grupos de observaciones que más se parecen entre sí y que a su vez se diferencian del resto.

TABLA I Clasificación de casas de acuerdo con su valor unitario $\left(\mathrm{UF} / \mathrm{m}^{2}\right)$

\begin{tabular}{|c|c|c|c|}
\hline \multirow{2}{*}{ TIPO } & $\begin{array}{c}\text { CANTIDAD DE } \\
\text { PROYECTOs* }\end{array}$ & UF/ $\mathbf{M}^{\mathbf{2}} \mathbf{M I N .}$ & UF/ $\mathbf{M}^{\mathbf{2}} \mathbf{\text { MAX. }}$ \\
\hline Casas A & 62 & 63,81 & 109,72 \\
\hline Casas B & 231 & 46,74 & 63,8 \\
\hline Casas C & 368 & 33,91 & 46,73 \\
\hline Casas D & 565 & 24,6 & 33,9 \\
\hline Casas E & 607 & 11,7 & 24,59 \\
\hline
\end{tabular}

* cada proyecto puede contener desde i hasta 500 unidades de vivienda unifamiliar

FUENTE ELABORACIÓN PROPIA CON BASE EN INFORMACIÓN DE INCITI Y COLLECT-GFK

\section{Grupos Socioeconómicos}

Considerando que es una variable relevante, se miden los tiempos de viaje a hogares según su Grupo Socioeconómico (GSE). Los GSE son una clasificación realizada por la empresa Adimark (2004) sobre la base de la información entregada por el Censo Nacional, en la cual se considera dos áreas fundamentales: posesiones del hogar y nivel de estudios del jefe de hogar. Según estos parámetros, se asigna un puntaje y se determina el GSE, que normalmente se agrupa en cinco segmentos: $A B C 1, C 2$, C3, D y E.

Para esta investigación se midió el tiempo de viaje desde cada proyecto inmobiliario hacia los hogares de cada GSE, de acuerdo con la metodología descrita en la sección siguiente. Se trabajó la información de GSE en distritos censales (mapas 2 a 7), pero estos fueron modificados, ya que en muchos distritos se incluían sectores urbanizados y sectores rurales indistintamente. Se separaron distritos y se les asignó población según información del Censo Nacional 2002 a nivel de manzanas, extraído mediante Redatam (programa para extraer datos censales). ${ }^{7}$

\section{Medición de tiempos de viaje}

El cálculo del tiempo de viaje para cada localización posible hacia un conjunto de destinos se basa en la metodología de análisis de superficies de costo (algoritmo implementado en diferentes programas de análisis espacial, como IDRISI, ${ }^{8}$ conocido como cost surface analysis). A continuación se describen los pasos seguidos para este cálculo:

1. El espacio de estudio es representado como una grilla (para este caso, con celdas de 500 x $500 \mathrm{~m}$.), en la cual cada celda tiene una impedancia o fricción, que representa el costo que implica atravesarla.

2. Para este estudio, el costo se refiere al tiempo que se requiere para atravesar la celda. Ese tiempo depende del tipo de vialidad presente en la celda.

7 En http://www.cepal.org/redatam/

8 En http://www.clarklabs.org/ 
3. Se cuenta con una cobertura de la vialidad en el área de estudio (se muestra en el mapa 1), la cual separamos en:

a) Autopistas: se les asigna una velocidad promedio de $80 \mathrm{~km} / \mathrm{h}$, lo cual implica que el tiempo de desplazamiento por la celda es de 0,375 minutos, considerando que las celdas tienen $500 \mathrm{~m}$ de lado.

b) Vialidad secundaria: se le asigna una velocidad promedio de $30 \mathrm{~km} / \mathrm{h}$, lo cual implica un tiempo de desplazamiento por cada celda de 1 minuto.

c) Sin vialidad: se le asigna una velocidad promedio de $5 \mathrm{~km} / \mathrm{h}$, lo cual implica un tiempo de desplazamiento por cada celda de 6 minutos.

4. Además se cuenta con una cobertura de pendientes en el área de estudio. Las pendientes están clasificadas en una escala de 1 a 5 , desde horizontal hasta acantilada. Para las zonas sin vialidad se multiplica el costo en tiempo por este valor de pendiente (por ejemplo, para las zonas horizontales se mantiene el mismo costo, ya que se multiplica $6 \mathrm{~min} \times 1=6 \mathrm{~min}$, pero para una zona de pendiente "moderada a fuerte", de valor 3 en la escala de pendientes, el costo es de 6 min x $3=18 \mathrm{~min}$ ).

5. Sobre la base de estos criterios se obtiene un mapa de costos.

6. El tiempo de desplazamiento entre todas las localizaciones y un destino se calcula con un algoritmo programado por los autores en Python. El algoritmo es similar al que usa el software IDRISI, en el cual se parte desde la celda destino y se van sumando los costos hacia las celdas vecinas en un avance que simula un derrame, en el cual se va recorriendo más rápidamente las celdas con menor costo o fricción, encontrando así el camino de menor costo a cada una de las celdas del área de estudio. La suma del costo para llegar a cada celda es un costo acumulado, que representa el tiempo de viaje hacia el destino para esa celda. Este algoritmo es una versión del algoritmo de Dijkstra para el cálculo de rutas de mínimo costo en redes (Dijkstra, 1959), pero aplicado a una grilla (por lo tanto, una red uniforme).

7. Se mide un tiempo de viaje promedio a diferentes destinos (todos los proyectos existentes de cierto tipo, o todos los hogares de cierto GSE), para lo cual se calcula el tiempo a cada uno de los destinos, y se obtiene un promedio ponderado. Lo anterior se alcanza de las siguientes formas:

a) Para proyectos: se multiplica el tiempo a cada proyecto (destino) por la misma cantidad de unidades del proyecto, luego se suman los tiempos multiplicados, y finalmente se divide por el total de unidades.

b) Para GSE: para este caso, se toma como destino el centroide de cada distrito censal y se multiplica el tiempo por la cantidad de hogares del GSE en cada distrito; luego se suman los tiempos multiplicados, y finalmente se divide por el total de hogares de ese GSE.

8. Para el caso de los tiempos de viaje a los proyectos, se midió el tiempo para cada año (considerando aquellos iniciados ese año y las autopistas existentes en ese mismo periodo). Con el fin de dar mayor relevancia a los proyectos más recientes -pero sin ignorar los posibles efectos de proyectos anteriores- el tiempo de viaje a otros proyectos se calcula como un promedio ponderado de los tiempos en años 
pasados, dando una mayor ponderación a los años más cercanos. Por ejemplo, para un proyecto del 2007, el tiempo de viaje a otros proyectos(t2007) viene dado por:

$$
t^{2007}=\frac{A \cdot t^{2006}+B \cdot t^{2005}+C \cdot t^{2004}}{A+B+C}
$$

Los valores de A, B y $\mathrm{C}$ van decreciendo a la mitad (en este caso $\mathrm{A}=1, \mathrm{~B}=0,5 \mathrm{y}$ $\mathrm{C}=0,25)$. Esta progresión podría ser calibrada para que los parámetros respondan al peso real de cada ańo en las decisiones de localización, pero para efectos de esta etapa se usa una progresión geométrica sencilla.

De esta forma, se obtiene para cada celda del área de estudio un tiempo promedio a la totalidad de casas nuevas de cierto tipo, o a la totalidad de hogares de algún GSE.

Esta medida de acceso se puede clasificar como de tipo gravitacional (Geurs \& Van Wee, 2004), ya que cumple con medir un costo asociado al desplazamiento a un conjunto de destinos, ponderado por la importancia o peso de esos destinos. Como se ve en la fórmula, no usa una curva exponencial de decaimiento en función de la distancia o tiempo, sino más bien lineal, ya que mantiene las unidades de tiempo (normalmente una medida de accesibilidad es inversa al tiempo: a mayor tiempo disminuye la accesibilidad y viceversa). El objetivo de este trabajo no se centra en desarrollar una medida de accesibilidad completa, sino más bien una aproximación que cumpla con informar sobre proximidades y separaciones entre localizaciones a una escala regional. Es por esto que la medida propuesta considera solo dos de los cuatro componentes que proponen Geurs y Van Wee (2004) para una medida de accesibilidad -el uso de suelo (localización de los destinos) y el sistema de transporte (tiempos por la red)-, y no aborda el factor temporal (disponibilidad de tiempo de las personas) e individual (disponibilidad de modos de transporte).

Por lo mismo, esta metodología mide tiempos de viaje simplificando la movilidad de las personas sobre la base de una velocidad promedio, solo diferenciada según el tipo de vía (autopistas, red vial secundaria y sectores sin red vial). No se considera que haya horas de mayor o menor congestión o el uso de transporte público. Para esta escala de análisis, y considerando que apunta a proyectos en áreas periurbanas, los supuestos señalados permiten conclusiones válidas, pero de todas formas los autores consideran a futuro integrar otras dimensiones o también usar modelos ya desarrollados, como estraus (De Cea, Fernández, Dekock, Soto \& Friesz, 2003).

\section{Interpretación de los resultados}

El análisis presentado sobre tiempos de viaje entre proyectos permite revelar algunos patrones de localización, aunque escapa a los objetivos de este texto el presentar una medida de segregación como tal. La inspección visual de los gráficos comparativos permite llegar a conclusiones, sin embargo, se mantiene como trabajo a futuro la confección de un índice que permita establecer comparaciones entre diferentes casos o cortes temporales. 


\section{Resultados}

A primera vista, en el mapa 1 se observa claramente cómo los proyectos se diferencian por precio, estableciéndose conos de desarrollo que siguen a los preexistentes en el área consolidada. Se muestran los Distritos Censales (2002) en escala de grises de acuerdo a la predominancia de Grupos Socioeconómicos, desde distritos con más de 5 veces más $\mathrm{ABC} 1$ y $\mathrm{C} 2$ que $\mathrm{D}$ y E, hasta distritos con una predominancia opuesta.

Luego, al estudiar los proyectos inmobiliarios individualmente, se advierte en cada uno un claro enfoque hacia un segmento específico de valor en el mercado. Los proyectos estudiados tienen un amplio rango de valor, entre los más económicos (585 uf) y los más caros (26.162 Uf) en el precio de las unidades. Sin embargo, la diferencia promedio entre la casa de menor y la de mayor precio, dentro de un proyecto, es de 1.316 uf (en promedio, la casa más cara de un proyecto es tan solo $39 \%$ más cara que la más barata).

Esta tendencia indica que la segregación residencial se reproduce en la menor escala, con la segmentación que hacen los proyectos, optando por atraer a un tipo de hogar específico más que buscar la mayor variedad de demanda posible.

\section{MAPA I Proyectos habitacionales en áreas suburbanas y perirubanas}

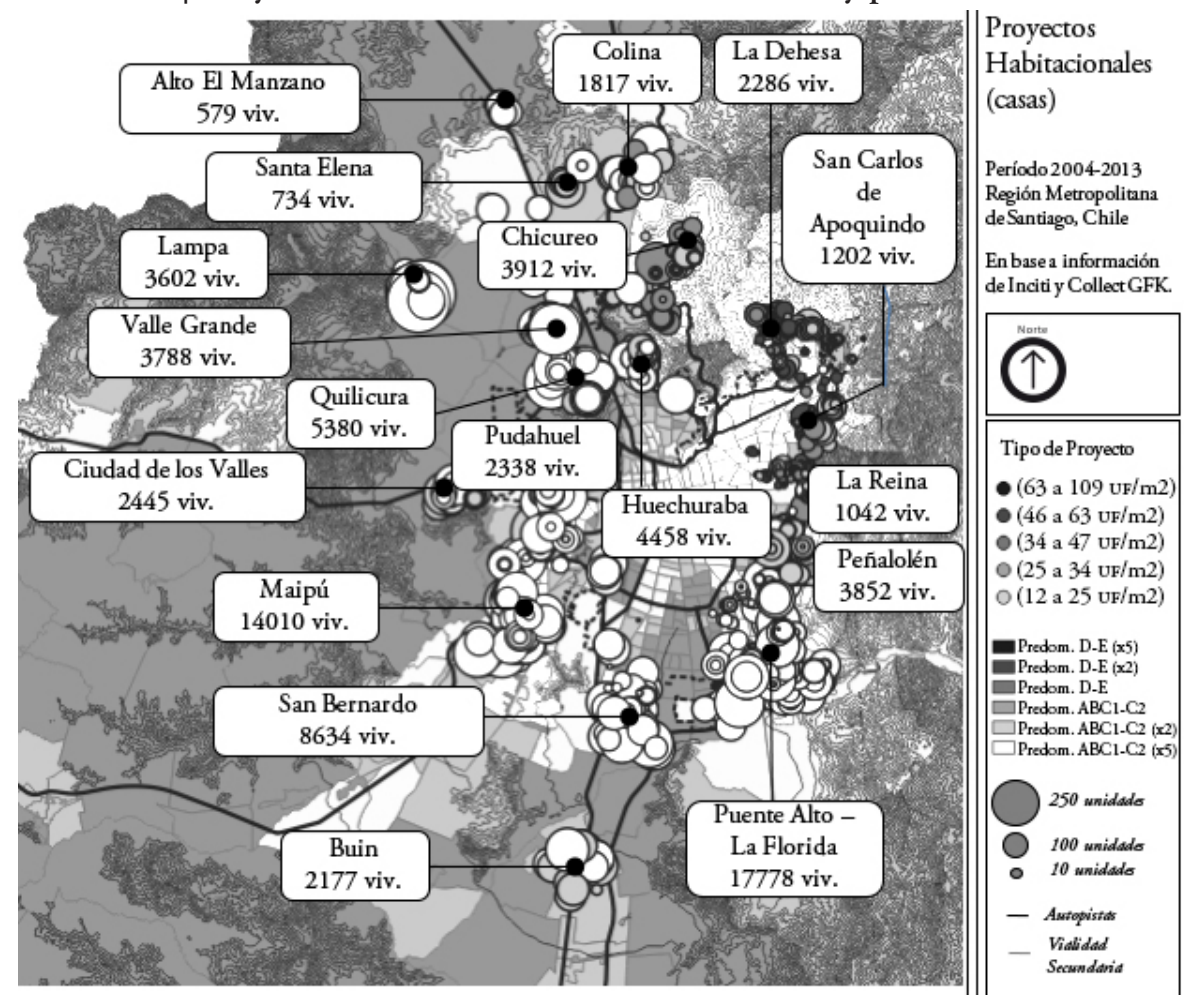

FUENTE ELABORACIÓN PROPIA CON BASE EN INFORMACIÓN DE INCITI Y COLLECT-GFK 
Por lo tanto, considerando que los proyectos tienen cierta uniformidad y los valores promedio de cada uno reflejan el valor de las unidades que se ofertan, se procedió a realizar un análisis univariado de la relación entre precio de los proyectos y las diferentes variables que se están trabajando.

En una primera apreciación de los sectores de desarrollo en la región, se observa que hay cuatro vectores de localización distinguibles:

1. Periferias extendidas: sectores en el sur poniente en los cuales se continúa el patrón de extendido de villas que se ha desarrollado en las últimas décadas, con estrecha relación a sectores de predominancia de GSE D y E. En este vector se encuentran los sectores de San Bernardo, Pudahuel, Maipú, Quilicura.

2. Periferias limitadas: sectores que, siendo periféricos, están limitados por la presencia de cerros, siendo continuación de áreas con predominancia $\mathrm{ABC} 1$ y C2, con una mayor cercanía a servicios. En este vector se encuentran los sectores de Peñalolén, La Reina y San Carlos de Apoquindo. Los desarrollos de La Florida y Puente Alto buscan esta situación como una continuación de Peñalolén, pero cercanos a grupos de menores ingresos. Lo mismo pasa con Huechuraba, pero buscando cierta cercanía con Vitacura.

3. Satélites en torno a localidades consolidadas: conjuntos exteriores a Santiago pero que se han desarrollado como extensión de localidades consolidadas, en entornos rurales y generalmente beneficiados por el paso de una autopista. En este vector se encuentran Buin, Lampa y Colina.

4. Satélites en faldeos de cerro: exteriores a Santiago, pero que no se adosan a ninguna localidad existente, sino más bien se alejan de estos subcentros buscando fondos de valles. En este vector se encuentran Chicureo, Santa Elena y Ciudad de los Valles.

Estos cuatro tipos de localización para desarrollos pueden diferenciarse por atributos relacionados con proximidades, tanto a densidad en general como a grupos socioeconómicos y proyectos preexistentes. A continuación se muestran los resultados de la medición de tiempos de viaje entre estos proyectos, clasificados por valor, y los hogares por Grupo Socioeconómico:

\section{Tiempos de viaje según Grupos Socioeconómicos (Censo 2002)}

De acuerdo con la metodología, se miden los tiempos de viaje promedio desde cada celda a hogares de cada grupo socioeconómico, lo cual muestra algunos patrones:

1. Básicamente, hay un patrón concéntrico para los cinco grupos, debido a que la mayor concentración está en el área urbana de Santiago, independientemente de dónde se concentren los grupos socioeconómicos.

2. Los GSE de mayores ingresos muestran menores tiempos de viaje en zonas específicas que los GSE de menores ingresos. Esto se debe a que los GSE de mayores ingresos se concentran en zonas específicas (cono de altos ingresos), a diferencia de los otros que se ubican en una zona más amplia de la ciudad. 
3. Se midió para todas las celdas un indicador que diera cuenta a la vez del tiempo de viaje a GSE de altos y de bajos ingresos, mediante la siguiente división:

$$
t_{i}=\frac{t_{A B C 1}+t_{C 2}}{t_{n}+t_{R}}
$$

donde $t_{\mathrm{x}}$ es el tiempo promedio desde la celda i hacia los hogares del GSE x.

\section{MAPA 2 Tiempos de viaje promedio a hogares según Grupo Socioeconómico}
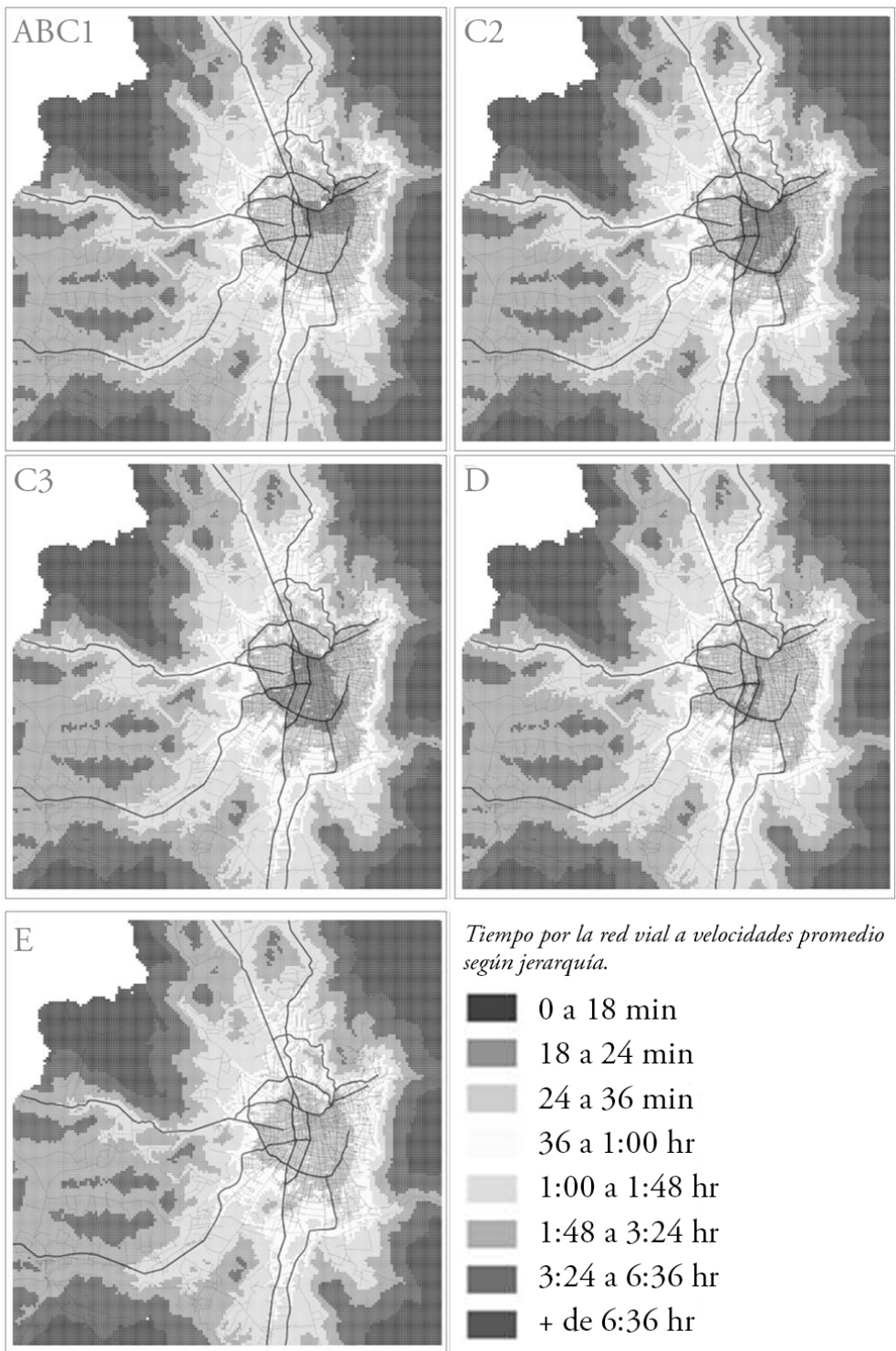

Tiempo por la red vial a velocidades promedio según jerarquí.
0 a $18 \mathrm{~min}$
18 a $24 \mathrm{~min}$
24 a $36 \mathrm{~min}$
36 a $1: 00 \mathrm{hr}$
$1: 00$ a $1: 48 \mathrm{hr}$
$1: 48$ a $3: 24 \mathrm{hr}$
$3: 24$ a $6: 36 \mathrm{hr}$
+ de $6: 36 \mathrm{hr}$

FUENTE ELABORACIÓN PROPIA CON BASE EN INFORMACIÓN DE INE (CENSO 2002) 
Una localización con un valor alto para este indicador significa que está lejos de GSE de altos ingresos y cerca de GSE de bajos ingresos. La espacialización de los resultados (mapa 3) muestra una diferenciación más clara de sectores, identificándose más claramente el cono de altos ingresos, pero también ejes de accesibilidad a GSE. Se aprecian elementos característicos del desarrollo de un sistema urbano:

1. Ejes: debido a que la red de autopistas produce áreas de mayor accesibilidad, cuando una de estas nace de un sector densamente poblado con cierta proporción de GSE y luego se interna en sectores rurales menos densos, se produce un eje de influencia de esos GSE sobre el área rural. Esto se observa para sectores como la ruta 68 hacia Valparaíso.

2. Espirales: se observa en el mapa 8 cómo los gSE altos de La Dehesa influyen sobre el desarrollo de Chicureo en la búsqueda de nuevo espacio libre, pero no en forma de eje sino como espiral, forma que es más eficiente, ya que no produce un mayor alejamiento de la ciudad (para este caso, el nuevo sector se conecta a los barrios altos, aunque también a sectores centrales a través de la autopista Los Libertadores).

3. Conos: se identifica un cono de influencia de GSE de menores ingresos hacia el sur poniente, tomando las comunas de Maipú, San Bernardo, Padre Hurtado, Peñaflor, Buin, etcétera.

Luego, si observamos el tiempo promedio de viaje a GSE de los diferentes tipos de proyectos (figura 1), encontramos una distribución que cumple con lo esperado. Los proyectos tipo A (de mayor precio) tienen tiempos de viaje reducidos a los GSE de mayores ingresos, y un tiempo creciente hacia los demás GSE. Los proyectos tipo B muestran un patrón similar, pero menos acentuado. Luego, los proyectos tipo C, D y E tienen un patrón similar entre ellos, lo cual acusa que los GsE de menores ingresos tienen una distribución más pareja en el territorio.

Para los proyectos tipo C, D y E, el mayor tiempo de viaje a hogares $\mathrm{ABC} 1$ y $\mathrm{E}$, y en menor medida a $\mathrm{C} 2$ y $\mathrm{D}$, se puede deber a la mayor concentración en áreas lejanas de los $\mathrm{ABC} 1$; y para el segundo caso, que los grupos E están dispersos a zonas periféricas.

Es relevante ver que el tiempo promedio a hogares en general va disminuyendo a medida que baja el valor del proyecto, lo que indica una mayor atracción a la densidad (o sectores más consolidados) para los proyectos de menor valor. 
MAPA 3 Proporción de tiempo de viaje promedio de grupos de altos ingresos y de bajos ingresos. En gris oscuro se muestran los sectores que tienen un bajo tiempo de viaje a GSE ABC1-C2 y alto tiempo de viaje a GSE D-E

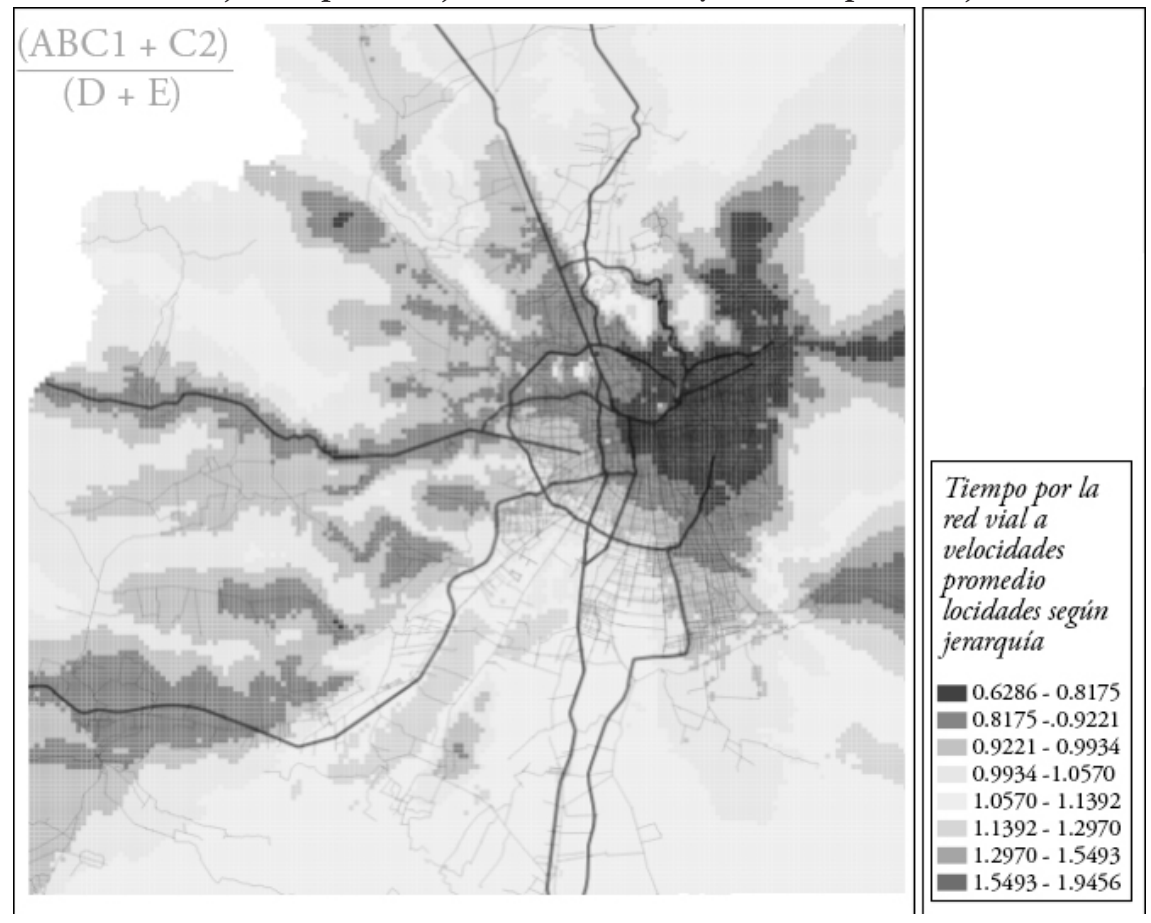

FUENTE ELABORACIÓN PROPIA CON BASE EN INFORMACIÓN DE INE (CENSO 2OO2)

FIGURA I | Tiempo de viaje promedio entre proyectos y hogares de diferentes Grupos Socioeconómicos

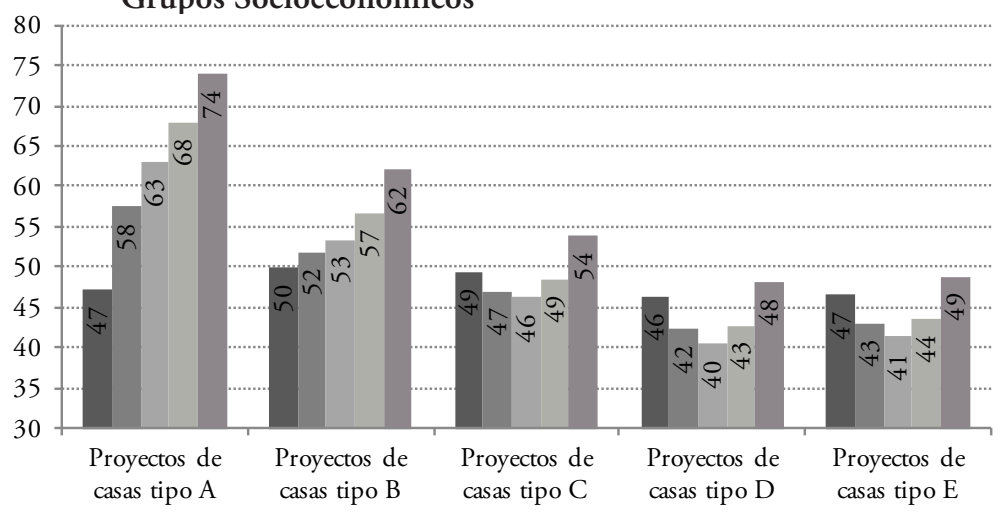

- Tiempo promedio a GSE ABC1 (min) -Tiempo promedio a GSE C2 (min)

-Tiempo promedio a GSE C3 (min) Tiempo promedio a GSE D (min)

- Tiempo promedio a GSE E (min)

FUENTE ELABORACIÓN PROPIA CON BASE EN INFORMACIÓN DE INCITI, COLLECT-GFK E INE (CENSO 2002) 
Tiempos de viaje entre tipos de proyectos

Para cada proyecto se calculó el tiempo promedio de viaje a los proyectos existentes hasta el año anterior al año de aparición de la etapa en estudio (figura 2).

El tiempo de viaje promedio a proyectos según tipo muestra un patrón bastante claro, siendo evidente la diferencia en el caso de los proyectos de mayor valor (tipo A), que tienen un tiempo promedio de 24 y 32 minutos a proyectos A y B en comparación a tiempos cercanos a 1 hora a los proyectos C, D y E. Es decir, los proyectos de mayor valor son los que más buscan estar cercanos a los de similar valor, lo que explica su concentración en ciertos lugares; y a la vez, son los que mayor distancia guardan respecto a los proyectos de menor valor.

Todos los tipos de proyectos presentan un menor tiempo hacia proyectos de su mismo tipo que hacia otros, salvo los tipo C, con una cercanía levemente mayor a los proyectos $\mathrm{D}$. La distribución observada es casi como un espejo con eje de simetría entre $\mathrm{B}$ y $\mathrm{C}$, lo que confirma que los proyectos tienden a estar entre similares y a distanciarse de los diferentes, ya sea por preferencias o por imposibilidad de localizarse en otros sectores.

La medición claramente indica una segregación, confirmándose las tendencias al respecto que se dan dentro del área consolidada.

FIGURA 2 Tiempo de viaje promedio entre proyectos y otros proyectos, por tipo

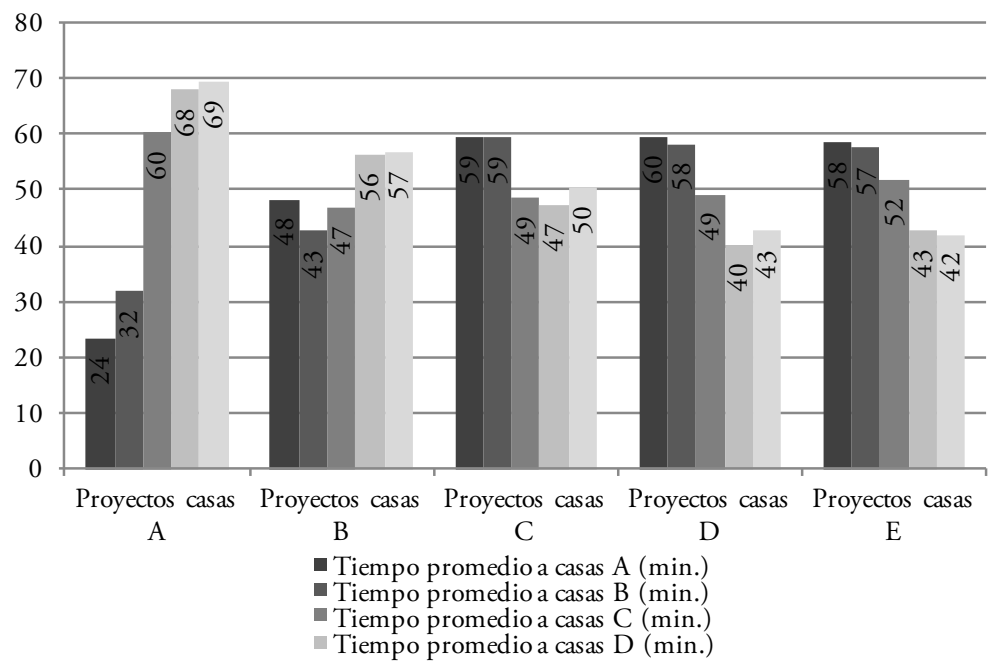

FUENTE ELABORACIÓN PROPIA CON BASE EN INFORMACIÓN DE INCITI, COLLECT-GFK E INE (CENSO 2OO2)

\section{Tiempos de viaje por sectores}

Con la base de proyectos disponible se separó por sectores identificables, con el objeto de estudiar cada uno de ellos a partir de las variables consideradas. Se observa que, en líneas generales, los sectores mantienen la tendencia descrita anteriormente. Como se representa en la figura 3, hay tres que se diferencian por su patrón de cercanía a proyectos de alto valor y lejanía a proyectos de bajo valor, como son La 
Dehesa, San Carlos de Apoquindo y La Reina, y que son justamente los sectores de mayor valor. En el extremo opuesto, hay un grupo ubicadoa en la periferia urbana de Santiago (Quilicura, Cerrillos, Maipú, Valle Grande, Pudahuel, San Bernardo, La Florida-Puente Alto y Lo Campino), con valor entre 20 y $25 \mathrm{uf} / \mathrm{m}^{2}$, y que comparten un mismo patrón de relativa lejanía a proyectos de alto valor y cercanía a los de bajo valor. Otro grupo de sectores identificables son los que han dado el llamado "salto de rana" (Alto El Manzano, Buin, Ciudad de los Valles-Lo Aguirre, Colina, Santa Elena y Chicureo), que con mayores tiempos de viaje en general tienen un patrón de cercanía a bajo valor y lejanía a alto valor, pero con un resultado en valor UF $/ \mathrm{m}^{2}$ superior a aquellos sectores más cercanos con similar patrón de cercanía a bajo valor y lejanía a alto valor.

FIgURA 3 Tiempo de viaje promedio a los tipos de proyectos por sector, comparado con el valor $\mathrm{UF} / \mathrm{m}^{2}$ promedio de los proyectos en el sector, y la densidad (hogares por hectárea)

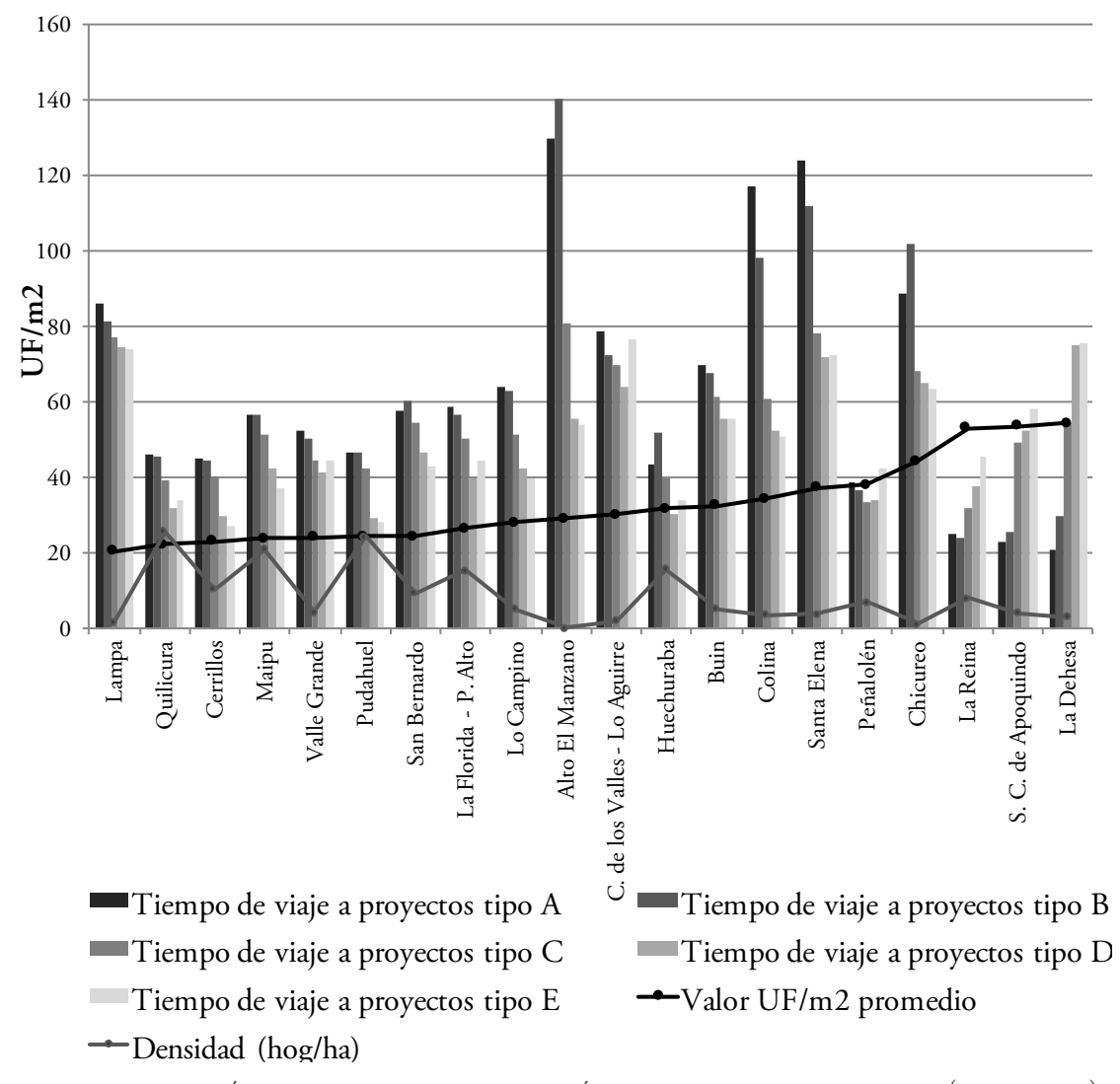

FUENTE ELABORACIÓN PROPIA EN BASE A INFORMACIÓN DE INCITI, COLLECT-GFK E INE (CENSO 2OO2) 
En la figura 3 se incluye la densidad en el distrito censal en que se ubica cada sector. Los con mayor valor son aquellos que están cercanos a alto valor y a su vez tienen baja densidad. Los sectores con alta densidad coinciden, en líneas generales, con aquellos de bajo valor.

Hay casos característicos aunque que no han sido muy explorados, como el sector de Buin (en el extremo sur en el mapa 1), que ha tenido un amplio desarrollo de proyectos de vivienda. A pesar de su entorno rural y baja densidad, presenta un tiempo de viaje a todos los tipos de proyectos muy inferior al de otros conjuntos que también son satélites a Santiago. Su conectividad por la ruta 5 y por el más reciente acceso sur permite esta condición.

El caso de Lampa es particular, ya que entra en el grupo de los sectores satélite, pero su valor promedio $\mathrm{UF} / \mathrm{m}^{2}$ es mucho menor que el observado en estas zonas. En este caso se aprecia la importancia de las autopistas, estando Lampa fuera del alcance de estas.

\section{Comentarios sobre la medición de proximidad entre proyectos}

Este trabajo tiene como hipótesis que el precio de un proyecto nuevo se explica por su relación con otros proyectos existentes. Y esta relación no se debe solo a que compartan la misma localización, sino a relaciones de proximidad que se establecen y que permiten asociar un sector con otro. Esto podría explicar el amplio desarrollo de viviendas de alto precio en sectores como Chicureo, asociado con Vitacura y La Dehesa, o el desarrollo de proyectos de menor precio en la periferia surponiente, que continúan con la expansión de villas para hogares de bajos ingresos en el sector desde hace décadas.

La investigación de relaciones de proximidad permite dar luces sobre casos como los de otros sectores que no se desarrollaron con la misma intensidad que Chicureo, como lo son Pirque o Calera de Tango, que pese a haber tenido un gran crecimiento de parcelas de agrado (sitios de $5.000 \mathrm{~m}^{2}$ con uso habitacional unifamiliar) para zonas de altos ingresos en los ańos noventa, no ofrecen el suficiente interés a los desarrolladores inmobiliarios como para impulsar figuras normativas del tipo de las zoduc (Zonas de Desarrollo Urbano Condicionado). Claramente no había condiciones de accesibilidad. Pirque, por ejemplo, queda en una suerte de cul-de-sac, a diferencia de Chicureo que se ubica en el camino hacia la ciudad de Los Andes y, por lo tanto, justifica una autopista como Los Libertadores. En Pirque, en cambio, sería necesario construir una autopista especialmente para los proyectos que ahí se desarrollaran, aumentando los costos, y también hay una mayor distancia a GSE de altos ingresos, interrumpida por sectores de bajos ingresos. Esto último no sucede en Chicureo, que mediante la construcción de autopistas (Radial Nororiente) y caminos (Juan Pablo II) salvó la interrupción que producían los cerros para vender proyectos como la "continuación natural" de sectores del cono de altos ingresos de la capital (por algo hay algunos que quieren llamar "Vitacura Norte" al sector de Chamisero en Chicureo, aludiendo a la comuna de altos ingresos a la que quieren asociarse.

Para medir las proximidades, en este trabajo se aborda el tiempo de viaje como la medida de relación entre sectores. Ello se calcula buscando la mayor verosimilitud 
respecto a los tiempos de viaje reales, a través de la velocidad promedio estimada para cada tipo de red vial. Esta medición se realiza pensando en que los hogares perciben los tiempos como una separación natural entre sectores. Los tiempos se miden en función de elementos que generan diferentes fricciones, siendo tales elementos, para este trabajo, las redes viales y la topografía.

Sin embargo, la forma en que las personas perciben la relación entre sectores tiene muchas otras dimensiones relativas a factores de referencia que forman límites y conexiones complejas de cuantificar. Pueden ser cerros, ríos, autopistas, valles, faldeos de cerro, que se conforman como elementos físicos reales o simbólicos que conectan o separan, como ya estudió Lynch (1960). Por otra parte, esas conexiones pueden ser discontinuas, como nodos de una red que conecta elementos de características similares.

La forma en que se puede medir este tipo de relaciones de forma sistemática es parte de los avances que se debe seguir para estudiar de mejor manera la forma en que se activan proyectos de diferentes tipos en el territorio.

\section{Conclusiones}

En resumen, en este estudio se desarrolló y aplicó una metodología destinada a medir dos variables relevantes para explicar la decisión de localización de proyectos inmobiliarios en el Área Metropolitana de Santiago: tiempo de viaje a otros proyectos y tiempo de viaje a grupos socioeconómicos específicos. A partir de tales cálculos se pudo identificar patrones espaciales de desarrollo en virtud de las relaciones entre tales proyectos.

Si bien la segregación por grupos socioeconómicos y la concentración de áreas de alto valor de suelo han sido estudiadas extensamente, el aporte de la metodología presentada es la posibilidad de medir tendencias de segregación entre grupos distantes o, más aún, las conexiones que existen en la región en cuanto a ejes de expansión de ciertos grupos socioeconómicos, como también bloqueos hacia este desarrollo por la presencia de grupos de menores ingresos, entre otros patrones dinámicos de segregación. De esta forma se puede explicar casos como el de Chicureo, cuyo éxito no tiene relación con la presencia anterior de grupos de altos ingresos en el sector, sino más bien con su proximidad en tiempos de viaje a otras localidades consolidadas de altos ingresos. Por otra parte, esta metodología puede explicar el hecho de que sectores como Pirque (al sur de la ciudad, con un desarrollo temprano -años noventa- para sectores de ingresos medio altos) no hayan estado sujetos posteriormente a proyectos más intensivos, como los de Chicureo. Desde esta perspectiva planteamos la hipótesis de que, en el caso de Pirque, la ausencia de desarrollo inmobiliario intensivo es producto de su desconexión con grupos de altos ingresos y cercanía a otros de bajos ingresos, como los presentes en Puente Alto.

En este sentido, la metodología presentada también permite entender el impacto que tiene la producción de nueva vialidad o sistemas de transporte en general en el atractivo que tienen algunas áreas. Se pueden así entender algunos proyectos como la Radial Nororiente y el camino Juan Pablo II, en tanto elementos que incentivan un desarrollo inmobiliario en extensión que, probablemente, no habría ocurrido 
de no existir estas vías que conectan el sector de altos ingresos de la ciudad con Chicureo. Por otra parte, esta metodología permite verificar ciertas relaciones de concentración y dispersión asimétricas entre los diferentes grupos. Los resultados muestran que tanto los proyectos de mayor precio como los GSE de altos ingresos se ubican concentrados en sectores específicos de la ciudad, lo cual reduce los tiempos de viaje entre ellos; y que por esto contrasta con los tiempos entre los proyectos de menor valor y GSE bajos, los cuales tienen un mayor tiempo de viaje entre similares, debido a que su distribución es más dispersa. Esta concentración hace que los GSE de mayores ingresos tengan una mayor facilidad para interactuar entre ellos y reproducir sus relaciones en el tiempo.

Este artículo presenta vínculos claros que se observan en cuanto a tiempos de viaje entre grupos; sin embargo, los orígenes de estas distribuciones son materia de discusión. No creemos que haya una simetría en las causas que determinan las proximidades entre grupos de altos ingresos y las que se dan entre aquellos bajos ingresos. Claramente los grupos de altos ingresos tienen mayor capacidad de decidir su localización, por lo que la proximidad entre ellos es una decisión acorde a sus preferencias; no así en el caso de grupos con menor capacidad de pago por vivienda, para los cuales la oferta se reduce a aquellos sectores con menor valor de suelo, generando una proximidad por descarte más que por opción directa. La cuantificación y modelamiento de las complejas relaciones de asimetrías en las preferencias, atracción y repulsión entre grupos, y su efecto sobre la ciudad (conos y espirales, por ejemplo), son campos en los cuales se podría investigar extensamente.

Si bien el objetivo de este artículo es presentar algunos atributos para explicar la localización residencial, la metodología usada también tiene potencial como medida de segregación, siendo más funcional que la usada tradicionalmente (basada en zonas), y también compatible con metodologías más recientes, como las revisadas. Sería interesante construir un indicador de segregación a partir de las mediciones presentadas y que pueda ser comparable en diferentes cortes temporales.

Consideramos interesante enfocar futuras investigaciones en segregación residencial en Santiago hacia la "experiencia de la segregación”, estudiando, por ejemplo, encuestas origen destino para entender este fenómeno no solo desde la localización residencial, sino también en función de la exposición a otros grupos durante los viajes a otros lugares (trabajo, educación), de acuerdo con el marco que presenta Kwan (2013).

Si bien en este artículo se presentan los desarrollos inmobiliarios estudiados como una especie de "ecología" de proyectos en la que hay decisiones de ubicación, para lo cual se toman en cuenta relaciones de proximidad y separación, no se puede dejar de mencionar que hay vectores de desarrollo que responden a políticas concertadas, ya sea desde la institucionalidad o desde las coaliciones entre privados y también público-privadas. En este sentido se destacan, por ejemplo, los sectores de Chicureo, al nororiente de la ciudad, que nace como una zona exclusiva a partir de modificaciones en la norma urbana, las cuales son ad hoc al surgimiento de conjuntos de proyectos de similares características.

En cuanto a la metodología de medición de accesibilidad de cada proyecto al resto de los mismos, y entre cada proyecto y las zonas de la ciudad, se optó por 
una medida sencilla e intuitiva, pero que no considera ciertas variables (principalmente congestión y disponibilidad de modos de viaje). La medición en forma de tiempos promedio es bastante clara en cuanto a las distancias en la ciudad, facilitando una noción de la separación real que existe entre grupos. Esto a diferencia de otros cálculos de accesibilidad, generalmente expresados en unidades de medida abstractas que son difíciles de interpretar para el lector no especializado. Consideramos que esto puede complementarse a futuro, aunque la medida usada cumple con informar proximidades y separaciones entre localizaciones de forma apropiada para una escala regional.

El análisis realizado se enmarca en un estudio más amplio de las tendencias de localización residencial y los atributos que las determinan, por lo que no se profundiza en este patrón observado como una medida o índice de segregación. Está claro que hay trabajos más exhaustivos en cuanto a segregación residencial en función de factores temporales, pero consideramos que el patrón presentado tiene valor como una medida sencilla aplicable a modelos de localización, o que por lo menos indica ciertos atributos importantes al momento de plantear un modelo. En este sentido, las variables exploradas son la base para un modelo de localización de oferta residencial en el espacio periurbano desarrollable en el marco de esta investigación, que permita entender las decisiones de desarrollo y, eventualmente, predecir tendencias de localización a futuro.

\section{Agradecimientos}

El presente artículo se enmarca en el contexto de la investigación "Modelo de localización de oferta habitacional en el espacio periurbano de Santiago" (Proyecto con Fondos FaU $\mathrm{N}^{\circ}$ 04/13), que busca producir un modelo que permita explicar y predecir tendencias de desarrollo de oferta habitacional en el espacio periurbano de Santiago. Los autores agradecen a Marcelo Bauzá, de Inciti, ${ }^{9}$ por su ayuda con la recolección de datos.

Los autores agradecen también el apoyo prestado por el Centro de Desarrollo Urbano Sustentable (cedeus) (Comisión Nacional de Investigación Científica y Tecnológica-CONicyt y Fondo de Financiamiento de Centros de Investigación en Áreas Prioritarias-FONDAP 15110020).

Agradecemos a dos revisores anónimos por sus valiosas observaciones, que permitieron enriquecer este trabajo significativamente. 


\section{Referencias bibliográficas}

Adimark (2004). Mapa socioeconómico de Chile: nivel socioeconómico de los hogares del país basado en datos del censo [s.n]. Chile: Autor. En hppt://bit.ly/laCqPia

Atkinson, R. \& Flint, J. (2004). Fortress uk? Gated communities, the spatial revolt of the elites and time-space trajectories of segregation. Housing Studies, 19 (6), 875-892. doi: $10.1080 / 0267303042000293982$

Bähr, J. \& Borsdorf, A. (2005). La ciudad latinoamericana. La construcción de un modelo. Vigencia y perspectivas. Urbe. Revista de ciudad, urbanismo y paisaje (Lima), 2 (2), $207-$ 222. En http://guzlop-editoras.com/web_des/arquit01/pld0472pdf.pdf

De Cea, J., Fernández, J. E., Dekock, V., Soto, A. \& Friesz, T. L. (2003, enero). estraus: A computer package for solving supply-demand equilibrium problems on multimodal urban transportation networks with multiple user classes. Presentado en 82nd Annual Meeting of Transportation Research Board (TRB), Washington, D.C., 12 al 16 de enero de 2003. En http://www.mctsoft.com/html/fr_mct5.htm

De Mattos, C. A. (1999). Santiago de Chile, globalización y expansión metropolitana: lo que existía sigue existiendo. EURE, 25 (76), 29-56. http://dx.doi.org/10.4067/S025071611999007600002

De Ramón, A. (2000). Santiago de Chile. Santiago: Sudamericana.

Dijkstra, E. W. (1959). A note on two problems in connexion with graphs. Numerische mathematik, 1 (1), 269-271. En http://www-m3.ma.tum.de/foswiki/pub/MN0506/ WebHome/dijkstra.pdf

Ducci, M. E. \& González, M. (2006). Anatomía de la expansión de Santiago, 1991-2000. En A. Galetovich (Ed.), Santiago. Dónde estamos y hacia dónde vamos (pp. 123-146). Santiago: Centro de Estudios Públicos (CEP).

Duncan, O. D. \& Duncan, B. (1955). A methodological analysis of segregation indexes. American Sociological Review, 20 (2), 210-217. En http://personal.psc.isr.umich.edu/ yuxie-web/files/demtech/Duncan_Duncan1955.pdf

Ellis, M., Wright, R. \& Parks, V. (2004). Work together, live apart? Geographies of racial and ethnic segregation at home and at work. Annals of the Association of American Geographers, 94 (3), 620-637. doi: 10.1111/j.1467-8306.2004.00417.x

Farber, S. \& Li, X. (2013). Urban sprawl and social interaction potential: an empirical analysis of large metropolitan regions in the United States. Journal of Transport Geography, 31, 267-277. http://dx.doi.org/10.1016/j.jtrangeo.2013.03.002

Figueroa, O. (2004). Infraestructura, servicios públicos y expansión urbana en Santiago. En C. de Mattos, M. E. Ducci, A. Rodríguez \& G. Yáñez, Santiago en la globalización: ¿una nueva ciudad? (pp. 243-272). Santiago: Ediciones SUR.

Geurs, K. T. \& Van Wee, B. (2004). Accessibility evaluation of land-use and transport strategies: Review and research directions. Journal of Transport geography, 12 (2), 127140. doi: $10.1016 /$ j.jtrangeo.2003.10.005

Hägerstraand, T. (1970). What about people in regional science? Papers in regional science, 24(1), 7-24. doi: 10.1111/j.1435-5597.1970.tb01464.x

Hannam, K., Sheller, M. \& Urry, J. (2006). Editorial: Mobilities, immobilities and moorings. Mobilities, 1 (1), 1-22. En http://www.lancaster.ac.uk/fass/projects/medmobilities/ docs/Editorial-Mobilities.pdf 
Hidalgo, R. (2004). De los pequeños condominios a la ciudad vallada: las urbanizaciones cerradas y la nueva geografía social en Santiago de Chile (1990-2000). EURE, 30 (91), 29-52. http://dx.doi.org/10.4067/S0250-71612004009100003

Hine, J. \& Mitchell, F. (2001). Better for everyone? Travel experiences and transport exclusion. Urban Studies, 38 (2), 319-332. doi: 10.1080/00420980020018619

Hurtubia, R. \& Bierlaire, M. (2014). Estimation of bid functions for location choice and price modeling with a latent variable approach. Networks and Spatial Economics, 14 (1), 47 65. doi: 10.1007/s11067-013-9200-z

Inostroza, L., Baur, R. \& Csaplovics, E. (2013). Urban sprawl and fragmentation in Latin America: A dynamic quantification and characterization of spatial patterns. Journal of environmental management, 115, 87-97. http://dx.doi.org/10.1016/j. jenvman.2012.11.007

Ioannides, Y. M. (2004). Neighborhood income distributions. Journal of Urban Economics, 56 (3), 435-457. En http://dx.doi.org/10.1016/j.jue.2004.04.006

Instituto Nacional de Estadísticas (INE), Chile. (2007). Ingresos de hogares y personas 2006. Santiago: Autor.

Jenks, G. F. (1967). The data model concept in statistical mapping. International yearbook of cartography, 7 (1), 186-190.

Kwan, M. P. (2013). Beyond space (As we knew it): Toward temporally integrated geographies of segregation, health, and accessibility. En M. P. Kwan, D. Richardson, D. Wang \& C. Zhou (Eds.), Space-Time Integration in Geography and GIScience (pp. 39-51). The Netherlands: Springer.

Leckie, G., Pillinger, R., Jones, K. \& Goldstein, H. (2012). Multilevel modeling of social segregation. Journal of Educational and Behavioral Statistics, 37 (1), 3-30. doi:10.3102/1076998610394367

Lee, B. A., Reardon, S. F., Firebaugh, G., Farrell, C. R., Matthews, S. A. \& O’Sullivan, D. (2008). Beyond the census tract: Patterns and determinants of racial segregation at multiple geographic scales. American Sociological Review, 73 (5), 766-791. doi: $10.1177 / 000312240807300504$

Lynch, K. (1960). The image of the city (Vol. 11, MIT Press Paperback Series). Cambridge, MA: MIT press.

Massey, D. S. \& Denton, N. A. (1988). The dimensions of residential segregation. Social Forces, 67(2), 281-315. doi: 10.2307/2579183

Martínez, F. (1996). MUSSA: Land use model for Santiago city. Transportation Research Record: Journal of the Transportation Research Board, 1552 (1), 126-134. doi: http://dx.doi. org/10.3141/1552-18

Palmer, J. R. (2013). Activity-space segregation: Understanding social divisions in space and time (Tesis doctoral, Universidad de Princeton, Princeton, NJ).

Rodríguez Vignoli, J. (2008). Movilidad cotidiana, desigualdad social y segregación residencial en cuatro metrópolis de América Latina. EURE, 34 (103), 49-71. En http://www.eure. cl/index.php/eure/article/view/1364

Rodríguez, Vignoli, J. (2012). ¿¿Policentrismo o ampliación de la centralidad histórica en el Área Metropolitana del Gran Santiago?: evidencia novedosa proveniente de la encuesta Casen 2009. EURE, 38 (114), 71-97. En http://www.eure.cl/index.php/eure/article/ view/205/557 
Sabatini, F. (2006). La segregación social del espacio en las ciudades de América Latina. Washington, D.C.: Inter-American Development Bank. En http://publications.iadb. org/handle/11319/5324

Sabatini, F., Cáceres, G. \& Cerda, J. (2001). Segregación residencial en las principales ciudades chilenas: tendencias de las tres últimas décadas y posibles cursos de acción. EURE, 27 (82), 21-42. En http://www.eure.cl/index.php/eure/article/view/1258

Schelling, T. (1978). Micromotives and macrobehavior. New York/London: W.W Norton.

Schnell, I. \& Yoav, B. (2001). The sociospatial isolation of agents in everyday life spaces as an aspect of segregation. Annals of the Association of American Geographers, 91 (4), 622636. doi: 10.1111/0004-5608.00262

Transsa Consultores (2013). Estudio de inserción en mercado, pDuc en Colina. No publicado.

Wang, D., Li, F. \& Chai, Y. (2012). Activity spaces and sociospatial segregation in Beijing. Urban Geography, 33 (2), 256-277. doi: 10.2747/0272-3638.33.2.256 
